\title{
Basic Calculus on Time Scale with Mathematica
}

\author{
Ahmet Yantır and Ünal Ufuktepe \\ Izmir Institute of Technology, Department of Mathematics , \\ Urla, Izmir, TURKEY \\ ahmetyantir@iyte.edu.tr \\ unalufuktepe@iyte.edu.tr \\ Communicated by Hidekazu Takahashi
}

\begin{abstract}
Mathematical modeling of time dependent systems are always interesting for applied mathematicians. First continuous and then discrete mathematical modeling are built during the mathematical development from ancient to the modern times. By the discovery of the time scales, the problem of irregular controlling of time dependent systems is solved in 1990's. In this paper, we explain the derivative of functions on time scales and the solutions of some basic calculus problems by using Mathematica.
\end{abstract}

\section{Introduction}

In order to unify results from calculus of real numbers Hilger and Aulbach generalized the definition of a derivative and integral to time scales, in order to create time scales calculus. [1] on the subject of time scales summarizes and organizes much of the time scale calculus. Other papers on time scales include joint and individual papers of Aragval, Bohner, Dosly, Erbe, and Hilcher.

In this paper, first we try to explain what time scales are. We give the definitions of time scales, forward and backward jump operators, and $\Delta$ derivative. We prove some theorems on $\Delta$ derivative and give some applications with Mathematica.

\section{What Is Time Scale}

Let $T$ be any closed subset of $\mathrm{R}$. We will denote a time scale by the symbol $T$.

Example 1. Thus the real numbers, the integers, the natural numbers, and the nonnegative integers are examples of time scales, as are

$\operatorname{In}[1]:=\mathrm{T} 1=0 \leq x \leq 1 \| 3 \leq x \leq 5$

$\operatorname{In}[2]:=\mathrm{T} 2=0 \leq x \leq 3\|x==4\| x==9$

$\operatorname{In}[3]:=\mathrm{T} 3=$ Table $\left[2^{n},\{n, 0,10\}\right]$

and the Cantor set while the rational, the irrational numbers, the complex numbers, and the open interval between 0 and 1 , are not time scales. $[1]$ 


\subsection{Forward Jump Operator}

Definition 1. Let $T$ be a time scale. For $t \in T$ we define define the forward jump operator $\sigma: T \rightarrow T$ by

$$
\sigma(t)=\operatorname{Inf}\{s: s \in T, s>t\}
$$

We define this operator in Mathematica as follows:

$\operatorname{In}[4]:=<<$ Algebra'InequalitySolve $^{6}$

$\operatorname{In}[5]:=$ Clear $[\operatorname{sigma}]$

$\operatorname{In}[6]:=\inf \left[\exp _{-}\right]:=\operatorname{Min}[\operatorname{Select}[\operatorname{Level}[\exp , 2]$, Element[\# , Reals] \&]];

$\operatorname{In}[7]:=\operatorname{sigma}\left[\mathrm{t}_{-}\right]:=\inf [$ InequalitySolve[T2 \&\& $\left.(x>t), \mathrm{x}]\right]$;

$\operatorname{In}[8]:=\operatorname{sigma}[1]$

Out $[8]:=1$

$\operatorname{In}[9]:=\operatorname{sigma}[3]$

Out $[9]:=4$

If $T=R$, then $\sigma(t)=t$

If $T=Z$, then $\sigma(t)=t+1$. Also $\sigma(\max T)=\max T$

Definition 2. $t$ is called right dense point if $\sigma(t)=t$, and right scattered if $\sigma(t)>t$.

\subsection{Backward Jump Operator}

Definition 3. For $t \in$ we define the backward jump operator $\rho: T \rightarrow T$ by

$$
\rho(t)=\sup \{s: s \in T, s<t\}
$$

We define this operator in Mathematica as follows:

$\operatorname{In}[10]:=$ Clear[rho,sup]

$\operatorname{In}[11]:=\sup [\exp ]:=\operatorname{Max}[\operatorname{Select}[\operatorname{Level}[\exp , 2]$, Element[\#, Reals] \&]];

$\operatorname{In}[12]:=\operatorname{rho}\left[\mathrm{t}_{-}\right]:=\sup [$ InequalitySolve[T2 \&\& $\left.(x<t), \mathrm{x}]\right]$;

$\operatorname{In}[13]:=\operatorname{rho}[1]$

Out $[13]:=1$

$\operatorname{In}[14]:=\operatorname{rho}[3]$

Out $[14]:=1$

If $T=R$, then $\rho(t)=t$.

If $T=Z$, then $\rho(t)=t-1$. Also $\rho(\min T)=\min T$ 


\subsection{Derivative on Time Scales}

We also need below the set $T^{k}$ which is derived from the time scale $T$ as follows:

$$
T^{k}=\left\{\begin{aligned}
T-\{\max T\} & , \quad \text { if } \max T<\infty \text { and } \max \mathrm{T} \text { is right scattered } \\
T, & \text { otherwise }
\end{aligned}\right.
$$

Definition 4. Let $f: T \rightarrow C$ and $t \in T^{k}$. If there exists a neighborhood $U_{t}$ such that

$$
|f(\sigma(t))-f(s)-a[\sigma(t)-s]| \leq \epsilon|\sigma(t)-s|
$$

is satisfied for all $t, a \in C$ and $s \in U_{t}$ then $f$ is $\Delta$ differentiable at the point $t$ and $a$ is called $\Delta$ derivative of $f$ at the point $t$.

$$
a=f^{\Delta}(t)=\lim _{s \rightarrow t} \frac{f(\sigma(t))-f(s)}{\sigma(t)-s}
$$

We defined $\Delta$ derivative on $T^{k}=T-\{\max T\}$. The question is" How can we define $\Delta$ derivative at the point $t=\max T$ ? $"$

$$
|f(\sigma(t))-f(s)-a[\sigma(t)-s]| \leq \epsilon|\sigma(t)-s|, \forall s \in U_{t}
$$

$t=\max T$ then a neighborhood $U_{t}$ of $t$ contains only $t$. So the derivative can be defined only at $t=s$,

$$
\begin{aligned}
|f(\sigma(t))-f(t)-a[\sigma(t)-t]| & \leq \epsilon|\sigma(t)-t| \\
|f(t)-f(t)-a[t-t]| & \leq \epsilon|t-t| \\
0 & \leq 0
\end{aligned}
$$

So the definition of $\Delta$ derivative is satisfied for every value of $a$. Then $a$ can not be determined uniquely.

Proposition 1. Let $t_{0} \neq \min T, \sigma\left(t_{0}\right)>t_{0}$ and $\rho\left(t_{0}\right)=t_{0}$ then the function $\sigma(t)$ has no $\Delta$ derivative at $t=t_{0}$

Proof: Assume that $a=\sigma^{\Delta}\left(t_{0}\right)$ then $\forall s \in U_{t}$

$$
\left|\sigma\left(\sigma\left(t_{0}\right)\right)-\sigma(s)-a\left[\sigma\left(t_{0}\right)-s\right]\right| \leq \epsilon\left|\sigma\left(t_{0}\right)-s\right|
$$

Case 1) Since $t_{0} \in U_{t_{0}}$ then we can take $s=t_{0}$ so for all $s \in U_{t_{0}}$

$$
\left|\sigma\left(\sigma\left(t_{0}\right)\right)-\sigma(s)-a\left[\sigma\left(t_{0}\right)-t_{0}\right]\right| \leq \epsilon\left|\sigma\left(t_{0}\right)-t_{0}\right|
$$

when we take the limit as $\epsilon \rightarrow 0$

$$
\begin{gathered}
\sigma\left(\sigma\left(t_{0}\right)\right)-\sigma\left(t_{0}\right)-a\left[\sigma\left(t_{0}\right)-t_{0}\right]=0 \\
a=\frac{\sigma\left(\sigma\left(t_{0}\right)\right)-\sigma\left(t_{0}\right)}{\sigma\left(t_{0}\right)-t_{0}} .
\end{gathered}
$$


Case2) Since $t_{0}$ is right scattered and left dense, the points $s \in U_{t_{0}}$ can be chosen from the left side of $t_{0}$. When $s \rightarrow t_{0}$ then $\sigma(s) \rightarrow t_{0} . \forall \epsilon>0$

$$
\begin{gathered}
\left|\sigma\left(\sigma\left(t_{0}\right)\right)-t_{0}-a\left[\sigma\left(t_{0}\right)-t_{0}\right]\right| \leq \epsilon\left|\sigma\left(t_{0}\right)-t_{0}\right| \\
\sigma\left(\sigma\left(t_{0}\right)\right)-\sigma\left(t_{0}\right)-a\left[\sigma\left(t_{0}\right)-t_{0}\right]=0 \\
a=\frac{\sigma\left(\sigma\left(t_{0}\right)\right)-\sigma\left(t_{0}\right)}{\sigma\left(t_{0}\right)-t_{0}}
\end{gathered}
$$

this is a contradiction.

Proposition 2. If $f: T \rightarrow C$ has a $\Delta$ derivative at $t \in T^{k}$ then $a=f^{\Delta}(t)$ is unique.

Proof: $\quad$ Let $a_{1}=f^{\Delta}(t)$ and $a_{2}=f^{\Delta}(t)$ then

$$
\begin{gathered}
\left|f(\sigma(t))-f(s)-a_{1}[\sigma(t)-s]\right| \leq \epsilon|\sigma(t)-s| \forall s \in U_{t}^{1}, \quad \forall \epsilon>0 \\
\left|f(\sigma(t))-f(s)-a_{2}[\sigma(t)-s]\right| \leq \epsilon|\sigma(t)-s|, \forall s \in U_{t}^{2}, \quad \forall \epsilon>0 .
\end{gathered}
$$

Let $U_{t}=U_{t}^{1} \cap U_{t}^{2}$ then both inequalities hold;

$$
\begin{gathered}
\left|\frac{f(\sigma(t))-f(s)}{\sigma(t)-s}-a_{1}\right| \leq \epsilon \quad \forall s \in U_{t}^{1}, \quad s \neq \sigma(t) \\
\left|\frac{f(\sigma(t))-f(s)}{\sigma(t)-s}-a_{2}\right| \leq \epsilon \quad, \forall s \in U_{t}^{2}, \quad s \neq \sigma(t)
\end{gathered}
$$

then for all $s \in U_{t},\left|a_{1}-a_{2}\right|=\left|a_{1}-a_{2}-\frac{f(\sigma(t))-f(s)}{\sigma(t)-s}+\frac{f(\sigma(t))-f(s)}{\sigma(t)-s}\right|$

$$
\begin{gathered}
\left|a_{1}-a_{2}\right| \leq\left|\frac{f(\sigma(t))-f(s)}{\sigma(t)-s}-a_{1}\right|+\left|\frac{f(\sigma(t))-f(s)}{\sigma(t)-s}-a_{2}\right| \\
\left|a_{1}-a_{2}\right|<\epsilon+\epsilon=2 \epsilon \\
a_{1}-a_{2}=0 \quad a_{1}=a_{2}
\end{gathered}
$$

Theorem 1. Let $f: T \rightarrow C$ and $t \in T^{k}$.

1) If $f$ has a $\Delta$ derivative at $t$ then $f$ is continuous at $t$.

2) If $f$ is continuous at $t$ and $t$ is right scattered then $f$ has a $\Delta$ derivative at $t$ and

$$
f^{\Delta}(t)=\frac{f(\sigma(t))-f(t)}{\sigma(t)-t} .
$$

3) If $t$ is right dense then $f$ has a $\Delta$ derivative at $t$ iff

$$
\lim _{s \rightarrow t} \frac{f(t)-f(s)}{t-s}
$$

is finite. Then 


$$
f^{\Delta}(t)=\lim _{s \rightarrow t} \frac{f(t)-f(s)}{t-s} .
$$

4) If $f(t)$ has a $\Delta$ derivative at $t$ then

$$
f(\sigma(t))=f(t)+f^{\Delta}(t) \cdot[\sigma(t)-t] .
$$

Lemma: [Continuity on $Z$ ] Let $E$ be a metric space and $x_{0} \in E$.

For given $\epsilon>0$ if there exists $\delta=\delta\left(\epsilon, x_{0}\right)$ such that

$$
d\left(f(x)-f\left(x_{0}\right)\right)<\epsilon
$$

For all $x \in U_{\delta}\left(x_{0}\right)=\left\{x \in E: d\left(x, x_{0}\right)<\delta\right\}$ then $\mathrm{f}$ is continuous at $x_{0}$.

Proof of the Lemma:

$$
d\left(x_{1}, x_{2}\right)=\left|x_{1}-x_{2}\right| \quad \forall x_{1}, x_{2} \in E=T .
$$

Let $T=Z$ and $x_{0} \in Z$, for all $\delta>0, \quad U_{\delta}\left(x_{0}\right)=\left\{x \in Z:\left|x-x_{0}\right|<\delta=x_{0}\right\}$

$$
\left|f(x)-f\left(x_{0}\right)\right|=\left|f\left(x_{0}\right)-f\left(x_{0}\right)\right|=0<\epsilon
$$

then every function defined on $Z$ is continuous.

\section{Proof:}

1) Since $\mathrm{f}$ has a $\Delta$ derivative at $\mathrm{t}$, for given $\epsilon>0$ there exist $U_{t}$ such that $\forall s \in U_{t}$

$$
\left|f(\sigma(t))-f(s)-f^{\Delta}(t) \cdot[\sigma(t)-s]\right| \leq \epsilon \cdot|\sigma(t)-s|
$$

then by using the triangle inequality, we have

$$
\begin{aligned}
|f(t)-f(s)| & \leq \epsilon \cdot|\sigma(t)-s|+\epsilon \cdot|\sigma(t)-t|+\left|f^{\Delta}(t)\right| \cdot|t-s| \\
& \leq \epsilon_{1}
\end{aligned}
$$

then $\mathrm{f}$ is continuous at $\mathrm{t}$, where $\epsilon_{1}=2 \cdot \epsilon \cdot|\sigma(t)-t|+\left|f^{\Delta}(t)\right|+\epsilon$

2)

$$
\begin{aligned}
\frac{f(\sigma(t))-f(s)}{\sigma(t)-s} & =\frac{f(\sigma(t))-f(t)+f(t)-f(s)}{\sigma(t)-s} \\
\lim _{s \rightarrow t} \frac{f(\sigma(t))-f(s)}{\sigma(t)-s} & =\lim _{s \rightarrow t} \frac{f(\sigma(t))-f(t)}{\sigma(t)-s}+\lim _{s \rightarrow t} \frac{f(t)-f(s)}{\sigma(t)-s}
\end{aligned}
$$

then

$$
f^{\Delta}(t)=\lim _{s \rightarrow t} \frac{f(\sigma(t))-f(t)}{\sigma(t)-s}
$$


3) $(\Rightarrow)$ Let $\mathrm{f}$ has a $\Delta$ derivative at $\mathrm{t}$ then

$$
\begin{gathered}
\left|f(\sigma(t))-f(s)-f^{\Delta}(t) \cdot[\sigma(t)-s]\right| \leq \epsilon \cdot|\sigma(t)-s|, \\
f^{\Delta}(t)=\lim _{s \rightarrow t} \frac{f(\sigma(t))-f(s)}{\sigma(t)-s} .
\end{gathered}
$$

Since $t$ is right dense then

$$
\lim _{s \rightarrow t} \frac{f(\sigma(t))-f(s)}{\sigma(t)-s}=\lim _{s \rightarrow t} \frac{f(t)-f(s)}{t-s}=f^{\Delta}(t)
$$

$(\Leftarrow)$ It is trivial.

4) Let $\sigma(t)=t$

$$
f(\sigma(t))=f(t)+f^{\Delta}(t) \cdot[\sigma(t)-t] \quad \Rightarrow f(t)=f(t)
$$

Let $\sigma(t)>t$

$$
\begin{aligned}
f^{\Delta}(t) & =\frac{f(\sigma(t))-f(t)}{\sigma(t)-t} \\
f(\sigma(t)) & =f(t)+f^{\Delta}(t) \cdot[\sigma(t)-t] .
\end{aligned}
$$

Theorem 2. Let $f, g: T \rightarrow C$ be functions and $t \in T^{k}$. If $f$ and $g$ have $\Delta$ derivative at $t$ then

1) $f+g$ has $\Delta$ derivative at $t$,

$$
(f+g)^{\Delta}(t)=f^{\Delta}(t)+g^{\Delta}(t)
$$

2) $k f$ has a $\Delta$ derivative at $t$ and $\forall k \in C$,

$$
(k \cdot f)^{\Delta}(t)=k \cdot f^{\Delta}(t)
$$

3) $f \cdot g$ has a $\Delta$ derivative at $t$,

$$
(f \cdot g)^{\Delta}(t)=f^{\Delta}(t) \cdot g(t)+f(\sigma(t)) \cdot g^{\Delta}(t)=f(t) \cdot g^{\Delta}(t)+f^{\Delta}(t) \cdot g(\sigma(t))
$$

4) If $g(t) \cdot g(\sigma(t) \neq 0))$ then $\frac{f}{g}$ has $\Delta$ derivative at $t$,

$$
\left(\frac{f}{g}\right)^{\Delta}(t)=\frac{f^{\Delta}(t) \cdot g(t)-f(t) \cdot g^{\Delta}(t)}{g(t) \cdot g(\sigma(t))}
$$




\section{Applications with Mathematica}

Example 2. Let $f(x)=x+1, h(x)=x^{2}$ is defined on

$T 4==5 \leq x \leq 10\|x==12\| x==18$. Find $\left(\frac{f}{h}\right)^{\Delta}(10)$.

Solution: By Definition 4

$\operatorname{In}[15]:=$ Clear $[\mathrm{T} 4, \mathrm{f}, \mathrm{s}]$

$\operatorname{In}[16]:=\mathrm{T} 4=5 \leq x \leq 10\|x==12\| x==18$;

$\operatorname{In}[17]:=f\left[x_{-}\right]:=x+1 ; h\left[x_{-}\right]:=x^{2}$

$\operatorname{In}[18]:=\operatorname{sigma}\left[\mathrm{t}_{-}\right]:=\inf [$ InequalitySolve[T4 \&\& $\left.(x>t), \mathrm{x}]\right]$;

$\operatorname{In}[19]:=\mathrm{s}=\operatorname{sigma}[10]$

Out[19]:= 12

$\operatorname{In}[20]:=\operatorname{Limit}[(\mathrm{f}[\mathrm{s}] / \mathrm{h}[\mathrm{s}]-\mathrm{f}[\mathrm{t}] / \mathrm{h}[\mathrm{t}]) /(\mathrm{s}-\mathrm{t}), \mathrm{t} \rightarrow 10]$

Out[20]: $=-\frac{71}{7200}$

So we get $\left(\frac{f}{h}\right)^{\Delta}(10)=-\frac{71}{7200}$. By Theorem 2 we can get the same result as follows

$\operatorname{In}[21]:=\mathrm{fd}=\operatorname{Limit}[(\mathrm{f}[\mathrm{s}]-\mathrm{f}[\mathrm{t}]) /(\mathrm{s}-\mathrm{t}), \mathrm{t} \rightarrow 10]$

Out $[21]:=1$

$\operatorname{In}[22]:=\mathrm{hd}=\operatorname{Limit}[(\mathrm{h}[\mathrm{s}]-\mathrm{h}[\mathrm{t}]) /(\mathrm{s}-\mathrm{t}), \mathrm{t} \rightarrow 10]$

Out $[22]:=22$

$\operatorname{In}[23]:=$ result $=\left(\mathrm{fd}^{*} \mathrm{~h}[10]-\mathrm{hd} * \mathrm{f}[10]\right) /(\mathrm{h}[\mathrm{s}] * \mathrm{~h}[10])$

Out[23]: $=-\frac{71}{7200}$

Example 3. Let $g(x)=x^{3}$ is defined on $T 5=\left\{2^{n}: n \in Z\right\} \bigcup\{0\}$. Find $g^{\Delta}(1)$.

Solution: $g$ is continuous by Lemma and $g^{\Delta}(t)=\frac{g(\sigma(t))-g(t)}{\sigma(t)-t}$ by Theorem 1 , then

$\operatorname{In}[24]:=\mathrm{T} 5=$ Table $\left[2^{n},\{n, 0,10\}\right]$;

$\operatorname{In}[25]:=\mathrm{g}\left[\mathrm{x}_{-}\right]:=x^{3}$;

$\operatorname{In}[26]:=\mathrm{T}=$ Or $@ @(x==\# \& / @ \mathrm{~T} 5)$;

$\operatorname{In}[27]:=\operatorname{sigma}\left[\mathrm{t}_{-}\right]:=\inf [$ InequalitySolve[T \&\& $\left.(x>t), \mathrm{x}]\right]$;

$\operatorname{In}[28]:=\mathrm{s}=\operatorname{sigma}[1] ; \mathrm{t}=1$;

$\operatorname{In}[29]:=(\mathrm{g}[\mathrm{s}]-\mathrm{g}[\mathrm{t}]) /(\mathrm{s}-\mathrm{t})$

Out $[29]:=7$

we get $g^{\Delta}(1)=7$.

\section{References}

1. Bohner,M., Peterson,A.: Dynamic Equations on Time Scales, Birkhäuser Boston, (2001)

2. Gray, J.W.: Mastering Mathematica, Academic Press, (1997)

3. Wolfram, S.: The Mathematica Book, Cambrigde Univ. Press, (1996) 